\title{
The Discovery of a P Cygni Analog in M31 ${ }^{1}$
}

\author{
Philip Massey ${ }^{2}$ \\ Lowell Observatory, 1400 W. Mars Hill Road, Flagstaff, AZ 86001 \\ Phil.Massey@lowell.edu
}

\begin{abstract}
We present spectroscopy and discuss the photometric history of a previously obscure star in M31. The spectrum of the star is an extremely close match to that of P Cygni, one of the archetypes of Luminous Blue Variables (LBVs). The star has not shown much variability over the past 40 years $(<0.2 \mathrm{mag})$, although small-scale (0.05 mag) variations over a year appear to be real. Nevertheless, the presence of a sub-arcsecond extension around the star is indicative of a past outburst, and from the nebula's size ( 0.5 pc diameter) we estimate the outburst took place roughly 2000 yrs ago. P Cygni itself exhibits a similar photometric behavior, and has a similar nebula ( $0.2 \mathrm{pc}$ diameter). We argue that this may be more typical behavior for LBVs than commonly assumed. The star's location in the HR diagram offers substantial support for stellar evolutionary models that include the effects of rotation, as the star is just at a juncture in the evolutionary track of a $85 M_{\odot}$ star. The star is likely in a transition from an $\mathrm{O}$ star to a late-type WN Wolf-Rayet.
\end{abstract}

Subject headings: stars: early-type — stars: evolution-stars: mass loss - supergiants

\section{Introduction}

Luminous Blue Variables (LBVs) are a rare class of luminous stars which undergo episodic mass-loss, and probably represent a transition between the most massive $\mathrm{O}$ stars and

\footnotetext{
${ }^{1}$ Based in part on observations made with the NASA/ESA Hubble Space Telescope, obtained from the Data Archive at the Space Telescope Science Institute, which is operated by the Association for Universities for Research in Astronomy (AURA), Inc., under NASA contract NAS 5-26555.

${ }^{2}$ Visiting Astronomer, Kitt Peak National Optical Astronomy Observatory, which is operated by AURA, Inc., under cooperative agreement with the National Science Foundation (NSF).
} 
the red supergiant and/or Wolf-Rayet stage. (See the recent conference proceedings edited by Davidson et al. 1988, Nota \& Lamers 1997, and de Groot \& Sterken 2001.) All of the "accepted" LBVs were discovered on the basis photometric and/or spectroscopic variability, but the number of LBV "candidates" (based upon spectroscopic similarities to known LBVs) exceeds this number (Parker 1997). Understanding the number of bona-fide LBVs relative to that of other evolved massive stars (Wolf-Rayets, red supergiants) in nearby galaxies (i.e., at differing metallicities) provides a crucial test of the success of modern stellar evolutionary theory (Maeder \& Meynet 2000). Massey (2002) has argued that if the archetypal LBVs P Cygni or $\eta$ Carina were located in M 31 or M 33 we would not know of them today, since their spectacular photometric outbursts were hundreds of years ago.

In this Letter we report the discovery of a star in M31 whose spectrum is uniquely similar to that of P Cygni itself. A comparison of the star's location with stellar evolutionary tracks provides a strong substantiation of the latest generation of stellar models that include the effects of rotation (Meynet \& Maeder 2005), while also offering a caution in interpreting evolutionary stages of these hydrostatic models. The star has been relatively photometrically stable for the past 40 years, yet it shows compelling evidence of an outburst 2 millennia ago. This provides further support for the argument large photometric variations on the time-scale of decades may be more a selection effect than a consequence of the physics of LBVs.

\section{The Discovery}

The Local Group Galaxies Survey (LGGS) project has obtained images of M31 with the Kitt Peak 4-m telescope. The LGGS has now completed the photometric calibrations and photometry for M31 (>350,000 stars), and is preparing the paper for publication. A subset of several dozen bright, blue stars were selected for spectroscopy with the WIYN ${ }^{1}$ 3.5-m telescope and Hydra multi-object fiber positioner. The selection criteria included $V<18, B-V<0.5$, and the reddening free index $Q=(U-B)-0.72(B-V)<-0.6$ in order to eliminate foreground dwarfs. The spectra were obtained using a $790 \mathrm{l} \mathrm{mm}^{-1}$ grating (KPC-18C) in second order, with a BG-39 blocking filter to filter out first order red light. The observations covered $3970 \AA$ to $5030 \AA$. The "blue" fiber bundle was used for these observations; each fiber is 3.1" in diameter. The detector was a Tektronix 2048 x 2048 device ("T2KA") with $24 \mu \mathrm{m}$ pixels. The resulting spectral resolution was $1.5 \AA$. Our exposure of the northern field (in which our object was found) consisted of six $30 \mathrm{~m}$ integrations (i.e., 3 hrs).

\footnotetext{
${ }^{1}$ The WIYN Observatory is a joint facility of the University of Wisconsin-Madison, Indiana University, Yale University, and NOAO.
} 
The field was reconfigured halfway through the sequence in order to update the apparent position changes due to refraction. The observation was made on (UT) 2005 September 29.

In reducing the data that night we were immediately struck by the similarity of one of the stars to the well-known Galactic star P Cygni. The following night we obtained a 10 s exposure of $\mathrm{P}$ Cygni itself with the same setup to confirm our memory. We give the comparison in Fig. 1.

The degree of similarity to P Cygni is nearly unique. While the term "P Cygni profile" (blue-shifted absorption with an emission component extending redwards from line center) is used to describe individual lines in other stars having this characteristic mass-loss signature, only a few stars show even a close resemblance to P Cygni itself throughout the blue-optical region, and all of these stars have been called LBVs. Even amongst these the differences can be striking. For instance, although the LMC LBV-candidate R81 has been described as the "P Cygni of the LMC" by Wolf et al. (1981a), its optical spectrum contains He I lines without a strong emission component; compare their Fig. 1 with our Fig. 1. Walborn (1989) describes other LBVs at minimum light (i.e., maximum $T_{\text {eff }}$ ) as having the same general spectral characteristics as $\mathrm{P}$ Cygni, but the comparison is more apt in some cases than in others. AG Car (Hutsemékers \& Kohoutek 1988, Fig. 1), AF And, M33 Var B (Kenyon \& Gallagher 1985, Figs. 2-3), and R127 (Stahl et al. 1983, Fig. 3) do all show the same general P Cygni lines in He I and in the Balmer lines as does P Cygni, but R71 is an another example where the He I lines do not show a pronounced emission component (Wolf et al. 1981b, Fig. 3). HDE 316285 also shows many similarities in the infrared (McGregor et al. 1988) and yellow-red (Morrison \& Rao (1990), but the blue-optical spectra shows little resemblance to P Cygni (Walborn \& Fitzpatrick 2000). In the Milky Way, the LBV He 3-519 (Smith et al. 1994) probably bears the most similar spectrum known to P Cygni.

Our M31 star is identified in the LGGS catalog as J004341.84+411112.0, based upon its J2000 coordinates (i.e., $\alpha=00^{\mathrm{h}} 43^{\mathrm{m}} 41.84, \delta=+41^{\circ} 11^{\prime} 12$. .' 0 ) determined from USNO-B astrometric positions in the field. It is located in A30, an OB association cataloged by van den Bergh (1964); see Fig. 2. We note with some amusement that one of the original five Hubble-Sandage variables (Hubble \& Sandage 1953), Var $19=$ AF And, is located just 2' away $^{2}$.

\footnotetext{
${ }^{2}$ We eliminated the possibility that we mistakenly observed AF And itself. First, the two stars are clearly distinct. Brian Skiff kindly compared our field to the finding chart of AF And (Variable 19) from Hubble (1929), the paper which first showed that M31 is a galaxy(!), and confirmed the J2000 coordinates from the Two Micron All Sky Survey (2Mass) as $\alpha=00^{\mathrm{h}} 43^{\mathrm{m}} 33 \mathrm{~s} 08, \delta=+41^{\circ} 12^{\prime} 10 . " 3$ (uncertainty $<0$." 2 ), which led to a revision by SIMBAD in their listed position by a few arcseconds. Second, we considered whether or not it was possible for the fiber positioner to have accidently dropped the fiber button, miraculously hitting
} 
The star was first cataloged (as "40 4631") by Berkhuijsen et al. (1988), who presented $U B V R_{J}$ photometry based upon photographic plates taken in 1963. The next published photometry is given by Magnier et al. (1992) from their 1990 BVRI CCD survey of a 1 $\operatorname{deg}^{2}$ field in M31, where it appears as object 228465. If the data are taken at face value, the star brightened by $0.6 \mathrm{mag}$ in $V$, and reddened by $0.5 \mathrm{mag}$ in $B-V$ during the 37 years (see Table 1). However, nearby $\left(<10^{\prime}\right)$ stars of a similar brightness show this same offset in $V$, so we discount this as an indication of variability. However, the photographic $B$ value of neighboring stars are more in accord with Magnier et al. (1992), albeit with large scatter. The LGGS CCD photometry, carried out in 2001-2002, agrees well with Magnier et al. (1992). The slight differences between our two observations (0.06 mag) appears to be real: the formal errors are one-tenth of that, and a comparison of the stars in overlap between the two fields indicate median differences $<0.01$ at all filters except $I$, for which there is a small systematic offset (0.02 mag) for the 2002 observation. G. Jacoby also kindly obtained current-epoch (2005 November) $V$ and $R$ images at WIYN for us, which we have calibrated using the LGGS photometry of stars of similar color. We conclude that over recent times the star has been relatively constant $(<0.2 \mathrm{mag})$ in brightness over the past 40 years, but that it does show real variations of order $0.05 \mathrm{mag}$. This behavior is quite similar to that of P Cygni, although different than many other LBVs (Israelian \& de Groot 1999).

Hill et al. (1995) identify their UV-bright source 30-3 with this star, using Magnier et al. (1992) as a cross-reference. They used Ultraviolet Imaging Telescope (UIT) data, and detected the star in the $2500 \AA$ image but not in the $1500 \AA$ image ${ }^{3}$. The fact that the star is UV-bright is consistent with the Massey et al. (1996) study of UIT sources in M33, which discovered many LBV candidates.

We checked the HST data archives for images of the star; only one WFPC2 image contains a (barely) unsaturated exposure. This is a 300 s exposure through the F675W ("R") filter, u42z5701r. The star is in fact slightly extended, with a full-width-at-half-maximum (fwhm) of 2.0 pixels $(0.2 ")$ of the WF3 chip. Other stars on the image have significantly

\footnotetext{
the position of $\mathrm{AF}$ And rather than our intended target. It was not. For one thing, we made a minor tweak in the positions of all of the fibers half way through the exposure (see above), and the spectrum before and after the reconfiguration matched. In addition, had the button been dropped the positioner would have been unable to retrieve it when we reconfigured for the next field, and that was not the case. We are indebted to Nolan Walborn for raising this particular specter, but we feel it has been completely exorcised.

${ }^{3}$ They also had ground-based $B$ and $R$ CCD photometry indicating a magnitude of $B=18.29$, which is about $0.3 \mathrm{mag}$ fainter at $B$ than what was seen by any of the other studies; they estimate their errors as 0.05-0.10 mag, but provide no details (date or telescope) of the observations. One can infer that the observation was likely made in the early 1990s, so similar in time to Magnier et al. (1992). They report $R=17.12$, which is in good agreement with the values found by Magnier et al. (1992) and the LGGS.
} 
smaller fwhms, as shown in Fig. 3. However, the star does not appear to be double, given the radial profile, just extended. If we make the simple assumption that the size of the image adds in quadrature, then there is something around the star that has a diameter of about 0." 015. At the distance of M31 (760 kpc, according to van den Bergh 2000), this would have a size of 0.5 pc. P Cygni is known to have several shells of gas around it; the most prominent of these has a diameter of 22 " ( $0.2 \mathrm{pc}$ ), which is taken as evidence that the star had a major mass-loss event 900 years ago (see discussion in Israelian \& de Groot 1999). If the expansion velocity is the same for our object, then the relevant time scale would be about 2,000 yr. Confirmation with the better scale of the ACS would be desirable; several ACS images in the archive include the field, but the star is saturated ${ }^{4}$.

Determining the star's absolute visual magnitude requires knowing the reddening. If the star's intrinsic color is the same as P Cygni's, $(B-V)_{0}=-0.19$, (Lamers et al. 1983), then $E(B-V)=0.64$. The foreground reddening to M31 is $E(B-V)=0.07$, but most of the reddening of OB stars in M31 is due to extinction within the disk of M31 (van den Bergh 2000). Four OB associations away from the minor axis were found to have $E(B-V)=0.12-0.24$ by Massey et al. (1986). The reddening across M31 is quite patchy, and A30's location is near the minor axis where inclination effects will result in a larger amount of dust through the line of sight, so the value $E(B-V)=0.64$ seems quite reasonable. We derive an absolute visual magnitude of $M_{V}=-8.9$. This may be compared to P Cygni's absolute visual magnitude, $M=-8.3$ (Lamers et al. 1983). If the effective temperatures (and hence bolometric corrections) are similar as well, as is implied by the strong spectral similarities, we can scale P Cygni's bolometric luminosity by 0.6 mag as well, and expect that our star has $L / L_{\odot}=10^{6.1}$. In Fig. 4 we show the location of the star in the H-R diagram along with the evolutionary tracks for $\mathrm{z}=0.04$ (corresponding to the metallicity of M31's HII regions according to Zaritsky et al. 1994) from Meynet \& Maeder (2005). According to their calculations, an object in this part of the H-R diagram would be spectroscopically identified as a late-type WN (WNL) Wolf-Rayet star, and should never undergo an LBV phase as such. However, Andre Maeder (private communication) emphasizes that since their calculations are hydrostatic one can not really tell what stage a star is in (LBV vs WNL) from the models, as the difference between the two may be primarily one of atmospheric extent. The star may be at a transition point, and may evolve to the WNL stage after episodes of mass loss. There are hints in the spectra that the star is nitrogen-rich (Fig. 1), as is P Cygni and many other LBVs (Walborn 1989); a better spectrum is needed for modeling the CNO abundances, and such work is planned.

\footnotetext{
${ }^{4}$ Our own scheduled parallel imaging of this region did not happen as a result of the failure of the Space Telescope Imaging Spectrograph.
} 


\section{Summary and Discussion}

The LGGS project has obtained photometry of >350,000 stars seen towards M31. One of the brightest of these, LGGS 004341.84+411112.0, turns out to have a spectrum that is remarkably similar to that of the P Cygni, one of the best studied LBVs in the Milky Way. The star has been relatively constant in $B$ over the past 40 years, with variations $<0.2 \mathrm{mag}$, but with evidence of variations of $0.05 \mathrm{mag}$ during a year. Much the same can be said for P Cygni (Israelian \& de Groot 1999). However, the star has likely had an outburst two millennia ago, as judged by the fact that the star is slightly extended HST images, indicative of a 0.5 pc nebula, similar to the 0.2 pc nebula seen around P Cygni. This has consequences for interpreting the nature of objects found with spectroscopic similarity to other known LBVs; these stars are considered LBV "candidates" until variability is established, but the present study emphasizes that this may require more than a lifetime.

If we assume that the spectral similarity of our star to that of P Cygni is indicative of a similar effective temperature (and hence intrinsic color and bolometric correction), we can place the star on the H-R diagram using our photometry. The star falls on the evolutionary track for a $85 M_{\odot}$ star at the extreme end its evolution to cooler temperature, just as the evolutionary tracks turns to lower luminosity (Meynet \& Maeder 2005). The star is likely in a transition between an $\mathrm{O}$ star and a WNL. This provides strong vindication of the current generation of stellar evolutionary models that include rotation, but it also notes the difficulties in interpreting the evolution phases implied by the models. An atmospheric analysis of the star is planned, and long-term follow-up (both photometric and spectroscopic) is warranted.

We are grateful for suggestions by an anonymous referee, as well as help from George Jacoby and Brian Skiff. Andre Maeder and Georges Meynet made very useful comments on the issue of the evolutionary status of this object. The spectroscopic observations were carried out with aid from Daryl Willmarth, Di Harmer, George Will, and Hillary Mathis. Parts of this project were funded by the NSF through grant 0093060, and by NASA through a grant (GO-9794) from the Space Telescope Science Institute, which is is operated by AURA, Inc., under NASA contract NAS 5-26555.

\section{REFERENCES}

Berkhuijsen, E. M., Humphreys, R. M., Ghigo, F. D., \& Zumach, W. 1988, A\&A, 192, 299

Davidson, K., Moffat, A. F. J., \& Lamers, H. J. G. L. M., Physics of Luminous Blue Variables 
(Dordrecht: Kluwer)

de Groot, M., \& Sterken, C. 2001, ASP Conf. Ser. 233, P Cygni 2000: 400 Years of Progress Gallagher, J. S. 1997, in Luminous Blue Variables: Massive Stars in Transition, ed. A. Nota \& H. J. G. L. M. Lamers (San Francisco: ASP), 389

Hill, J. K. et al. 1995, ApJS, 98, 595

Hodge, P. W. 1981, Atlas of the Andromeda Galaxy (Seattle: Univ of Washington Press)

Hubble, E. P. 1929, ApJ 69, 103

Hubble, E., \& Sandage, A. 1953, ApJ, 118, 353

Hutsemékers, D., \& Kohoutek, L. 1988, A\&AS, 73, 217

Israelian, G., \& de Groot, M. 1999, SSRv, 90, 493

Kenyon, S. J., \& Gallagher, J. S. 1985, ApJ, 290, 542

Lamers, H. J. G. L. M., de Groot, M., \& Cassatella, A. 1983, A\&A, 128, 299

McGregor, P. J., Hyland, A. R., \& Hillier, D. J. 1988, ApJ, 324, 1071

Magnier, E. A., Lewin, W. H. G., van Pardijs, J., Hasinger, G., Jain, A., Pietsch, W., Trümper, J. 1992, A\&AS, 96, 379

Massey, P. 2003, ARA\&A, 41, 15

Massey, P., Armandroff, T. E., \& Conti, P. S. 1986, AJ, 92, 1303

Massey, P., Bianchi, L., Hutchings, J. B., \& Stecher, T. P. 1996, ApJ, 469, 629

Maeder, A. \& Meynet, G. 2000, A\&A, 361, 159

Meynet, G., \& Maeder, A. 2005, A\&A, 429, 581

Morrison, N. D., Rao, S. M. 1990, in Properties of Hot Luminous Stars, ed. C. D. Garmany (San Francisco: ASP), 53

Nota, A., \& Lamers, J. G. L. M., ASP Conf. Ser. 120, Luminous Blue Variables: Massive Stars in Transition (San Francisco: ASP)

Parker, J. W. 1997, in ASP Conf. Ser. 120, Luminous Blue Variables: Massive stars in Transition (San Francisco: ASP), 368

Smith, L. J., Crowther, P. A., \& Prinja, R. K. 1994, A\&A, 281, 833

Stahl, O., Mandel, H., Wolf, B., Gäng, Th., Kaufer, A., Kneer, R., Szeifert, Th., \& Shao, F. 1993, A\&AS, 99, 167

Stahl, O., Wolf, B., Klare, G., Cassatella, A., Krautter, J., Persi, P., Ferrari-Toniolo, M. 1983, A\&A, 127, 49 
van den Bergh, S. 1964, ApJS, 9, 65

van den Bergh, S. 2000, The Galaxies of the Local Group (Cambridge: University Press), 44

Walborn, N. R. 1989, in Physics of Luminous Blue Variables, ed. K. Davidson, A. f. J. Moffat, \& H. J. G. L. M. Lamers (Dordrect: Kluwer), 27

Walborn, N. R. \& Fitzpatrick, E. L. 2000, PASP, 112, 50

Wolf, B., Appenzeller, I., \& Stahl, O. 1981b, A\&A, 103, 94

Wolf, B., Stahl, O., de Groot, M. J. H., \& Sterken, C. 1981a, A\&A, 99, 351

Zaritsky, D., Kennicutt, R. C., \& Huchra, J. P. 1994, ApJ, 420, 87 
Table 1. Photometry of LGGS 004341.84+411112.0

\begin{tabular}{|c|c|c|c|c|c|c|c|c|c|c|c|}
\hline Date & $V$ & $\sigma_{V}$ & $B-V$ & $\sigma_{B-V}$ & $U-B$ & $\sigma_{U-B}$ & $V-R^{\mathrm{a}}$ & $\sigma_{V-R}$ & $R-I^{\mathrm{a}}$ & $\sigma_{R-I}$ & Ref/ID ${ }^{b}$ \\
\hline 1963 Aug/Sep & 18.13 & 0.19 & -0.14 & 0.29 & -0.31 & 0.26 & $\ldots \mathrm{c}$ & $\ldots \mathrm{c}$ & $\ldots$ & $\ldots$ & [BHG88] 404631 \\
\hline 1990 Sep $12-27$ & 17.531 & 0.061 & 0.407 & 0.088 & $\ldots$ & $\cdots$ & 0.408 & 0.097 & 0.229 & 0.086 & [MLV92] 228465 \\
\hline 2001 Sep 20-22 & 17.583 & 0.004 & 0.432 & 0.005 & -0.750 & 0.004 & 0.387 & 0.006 & 0.222 & 0.006 & LGGS $004341.84+411112.0$ \\
\hline 2002 Sep 11 & 17.511 & 0.005 & 0.478 & 0.005 & -0.771 & 0.005 & 0.421 & 0.005 & $0.332:$ & 0.005 & LGGS $004341.84+411112.0$ \\
\hline 2005 Nov 7 & 17.458 & 0.005 & $\ldots$ & $\ldots$ & $\ldots$ & $\ldots$ & 0.411 & 0.005 & $\ldots$ & $\ldots$ & New \\
\hline
\end{tabular}

${ }^{\text {a }} R$ and $I$ are on the Kron-Cousins system.

${ }^{\mathrm{b}}$ BHG88=Berkhuijsen et al. 1988; MLV92=Magnier et al. 1992; LGGS=Massey et al. in prep.

${ }^{\mathrm{c}}$ Berkhuijsen et al. 1988 do report $R$ photometry, but it appears to be on the Johnson rather than Kron-Cousins system. 


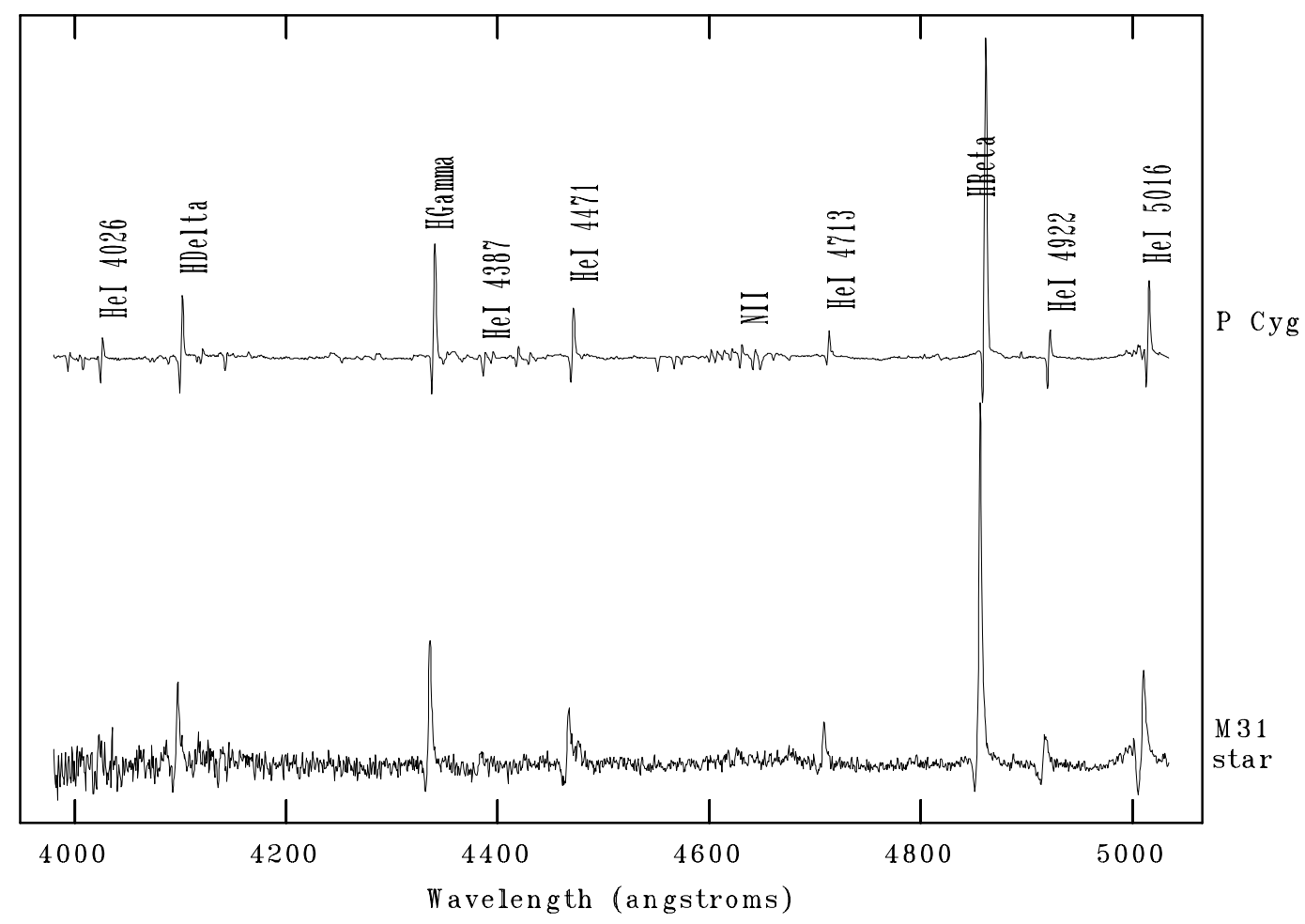

Fig. 1.- Comparison of the spectrum of LGGS J004341.84+411112.0 with P Cygni. Major lines have been identified; see also Stahl et al. (1993) 


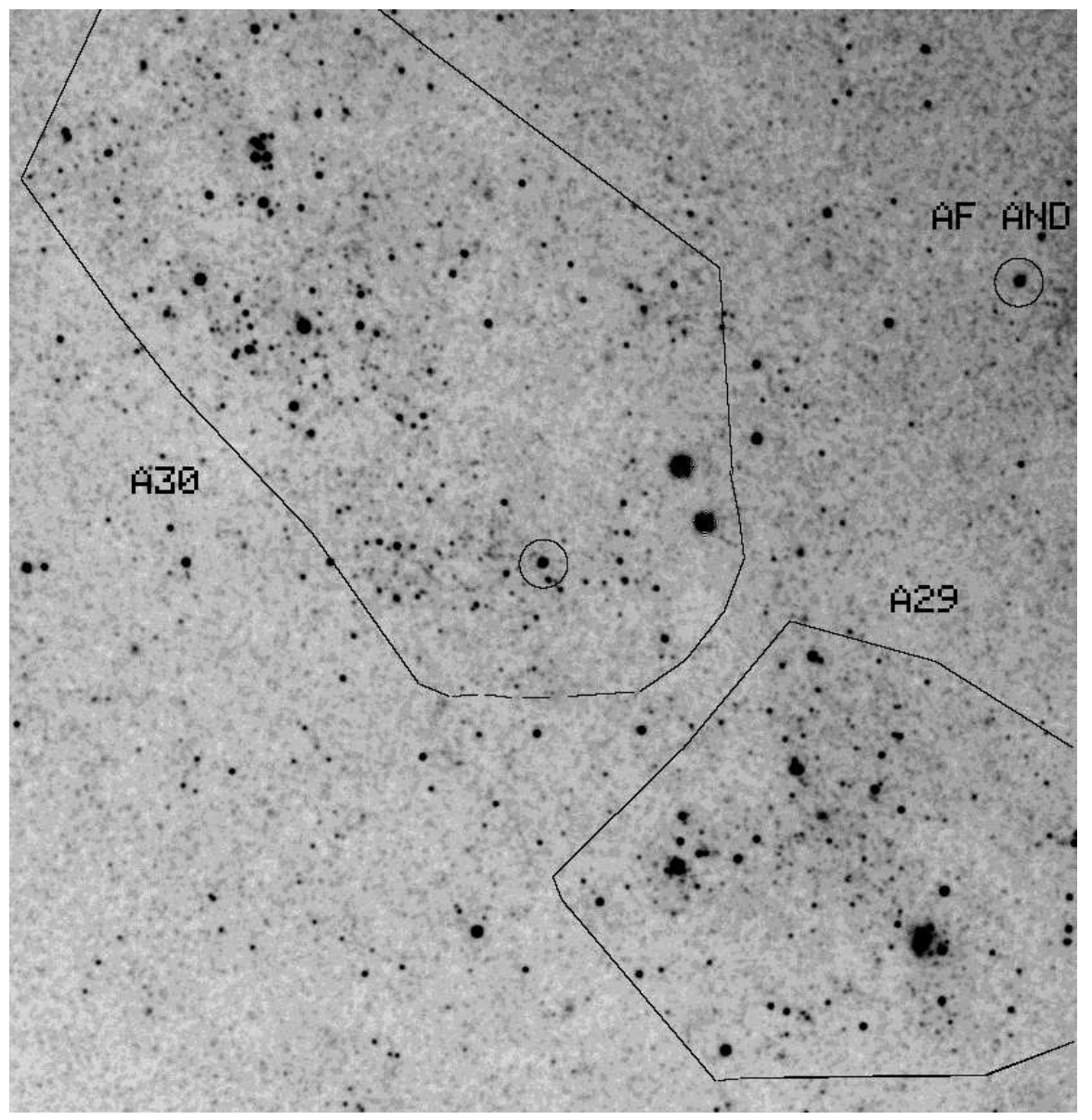

Fig. 2. - The location of LGGS J004341.84+41112.0. This star circled in the middle of this $V$ image (from the LGGS) is the newly found P Cygni analog. The well-known LBV AF And is located 2' to the NW. The circles have a diameter of 10". The outlines of the two OB associations A30 and A29 (van den Bergh 1964), based upon Hodge (1981). 


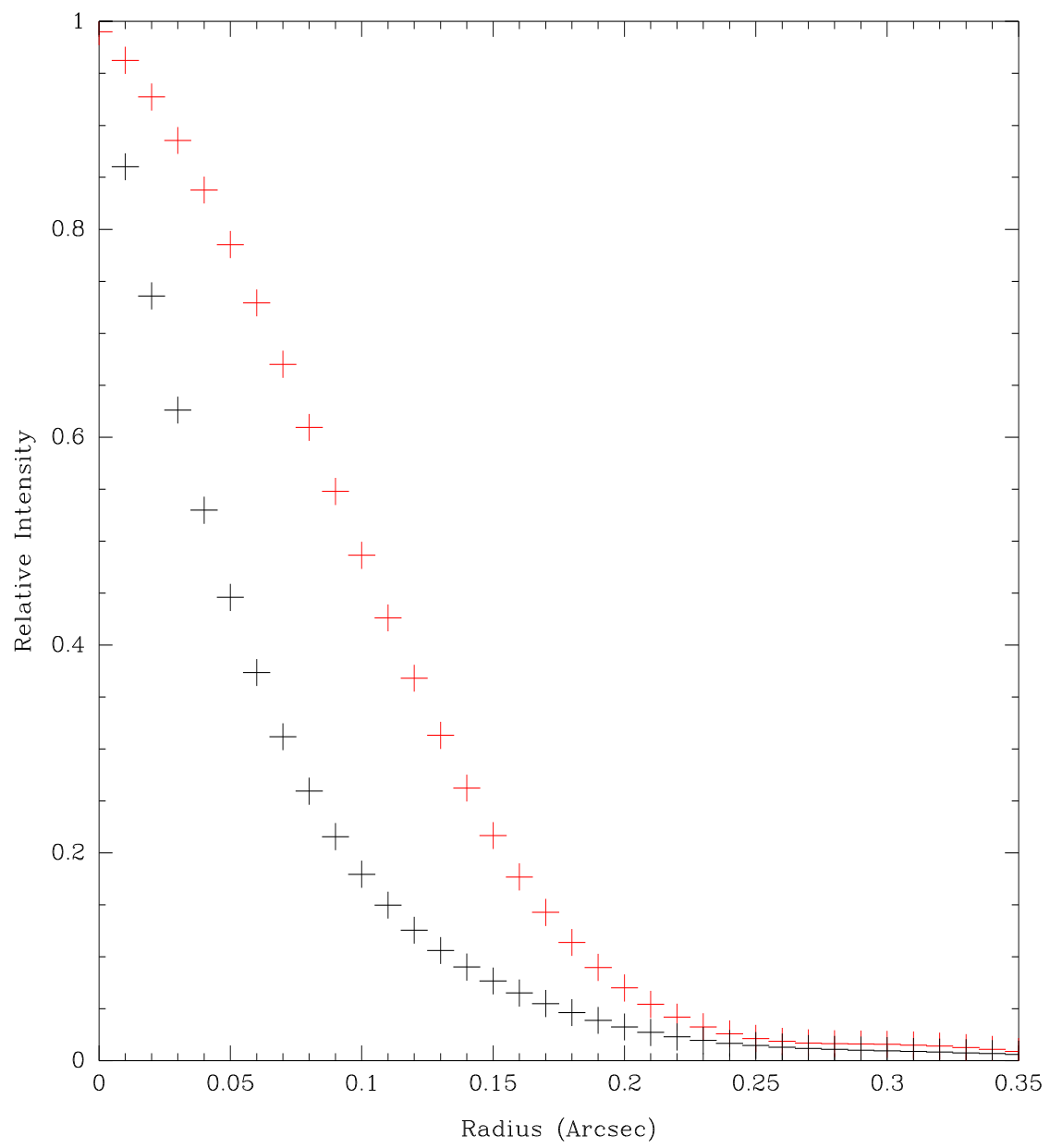

Fig. 3.- Spatial extension of LGGS J004341.84+411112.0. The radial profile of J004341.84+4111112.0 was measured from a WFPC2 image (WF3 chip), and is shown in red. The profile of another star on the same WF3 image is shown in black for comparison. 


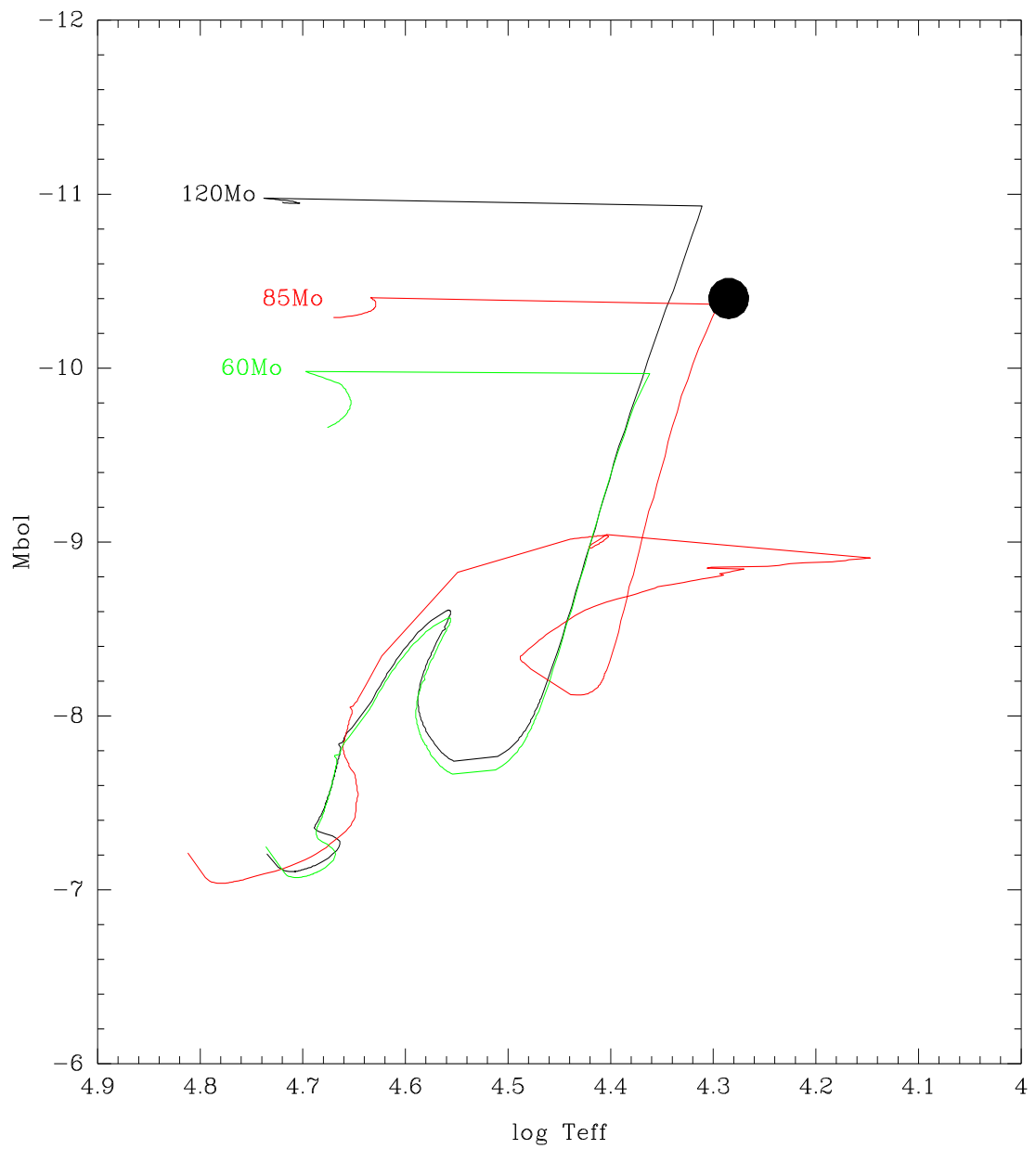

Fig. 4.- . The location of M31's P Cygni analog in the H-R diagram. We have plotted the location of LGGS J004341.84+411112.0 as a black dot, where we have assumed that its bolometric correction and effective temperatures are the same as those of P Cygni (Lamers et al. 1983). The evolutionary tracks of Meynet \& Maeder (2005) for an initial rotation velocity of $300 \mathrm{~km} \mathrm{~s}^{-1}$ are shown as solid colored lines, with the initial masses indicated. The star would appear to be at the coolest point in the evolution of a $85 M_{\odot}$ star, likely a transition point between an $\mathrm{O}$ star and a Wolf-Rayet. 